\title{
The use of Computer Guided Implant Surgery in Oral Rehabilitation: A Literature Review
}

\author{
${ }^{1}$ Vinicius Ibiapina Mascarenhas, ${ }^{2}$ Rafael Scaf de Molon, ${ }^{3}$ Lívia Jacovassi Tavares, ${ }^{4}$ Lara Maria Ferreira Mendes \\ ${ }^{5}$ Mateus Rodrigues Tonetto, ${ }^{6}$ Matheus Coelho Bandeca
}

\begin{abstract}
The virtual planning of dental implants is a technology that brings many benefits to practitioners and patients who undergo a prosthetic rehabilitation. The cone beam computed tomography (CBCT) produces high-resolution images allowing to implant a breakthrough in preoperative planning, making planning more accurate. The virtually guided surgery is a surgery planned based computers in a 3D anatomical model of the patient and transferred to the surgical procedure through guides built especially for this purpose. The objective of this study is to report the current concepts in the literature on virtually guided surgery, emphasizing its applicability, indications and benefits in prosthetic rehabilitation with dental implants. Thus, it was concluded that the technique of guided surgery represents an advance in the field of implantology significantly decreasing errors, bringing good results postoperative and increasing predictability of the results, one technique suitable for various cases.
\end{abstract}

Keywords: Dental implant, Image guided surgery, Dental prostheses.

How to cite this article: Mascarenhas VI, de Molon RS, Tavares LJ , Mendes LMF, Tonetto MR, Bandeca MC. The use of Computer Guided Implant Surgery in Oral Rehabilitation: A Literature Review. World J Dent 2014;5(1):60-63.

\section{Source of support $\mathrm{Nil}$}

Conflict of interest None

\section{INTRODUCTION}

The guided surgery based on three-dimensional (3D) computed images allows a better evaluation, bony

\footnotetext{
${ }^{1,3}$ MSC Student, ${ }^{2,5} \mathrm{PhD}$ Student, ${ }^{4}$ DDS Student, ${ }^{6} \mathrm{Head}$ of Postgraduation

${ }^{1,2}$ Department of Diagnosis and Surgery, School of Dentistry at Araraquara, Universidade Estadual Paulista, São Paulo, Brazil

${ }^{3}$ Department of Dental Materials and Prosthodontics, School of Dentistry at Araraquara, Universidade Estadual Paulista, São Paulo, Brazil

${ }^{4} S$ chool of Dentistry, Universidade Federal do Piaui, Teresina Piaui, Brazil

${ }^{5}$ Department of Restorative Dentistry, School of Dentistry at Araraquara, Universidade Estadual Paulista, São Paulo, Brazil

${ }^{6}$ Department of Dentistry, CEUMA University, São Luis, Brazil

Corresponding Author: Rafael Scaf de Molon, PhD Student Department of Diagnosis and Surgery, School of Dentistry at Araraquara Universidade Estadual Paulista; RuaHumaitá, 1680 14801-903, Araraquara, São Paulo, Brazil, Phone: +55 (16) 31141247,e-mail: molon.foar@yahoo.com.br
}

anatomy visualization, prosthetic planning and greater treatment outcomes predictability in oral rehabilitation with dental implants. The conventional techniques for preclinical assessment, such as visualization, pal pation and two-dimensional (2D) radiographs are being complemented with the development of a technology based in computerized three-dimensional structures planning. Cone beam computed tomography (CBCT) allow more accurate images and this technology can be obtained with a lower radiation dose compared to the conventional tomographys. A fter scanning, the images are transferred to a specific software allowing work with $3 \mathrm{D}$ anatomical structures identical to the original anatomy of the patient. CBCT allowing make prior surgery planning in regard to prosthetic and anatomical aspects and thus, being more accurate. ${ }^{1,2}$

The guided surgery is a computerized technology that brings many benefits to the professionals and patients. Some benefits to the guided surgery can be mentioned: possibility to flapless surgery, decreased in surgical time, reduction in the inflammatory response, better wound healing, decreased pain and discomfort in the immediate postoperative period, and immediate provisionalization of the prosthesis with highly precision, bringing a more predictable and accurate result. ${ }^{3,4}$

The aim of this study was to describe and discuss the current concepts on computed guided surgery, emphasizing its applicability, indications and benefits in prosthetic rehabilitation with dental implants.

\section{LITERATURE REVIEW}

The importance of computed tomography (CT) in the late 1980s to the imaging sciences and diagnostic of maxillary bones became more relevant with the introduction of high-resolution CT. Evaluation of dento-osseous lesions, maxillofacial deformities and temporomandibular joint (TMJ) access were the initial goal of CTs, but al so became effective for evaluation and diagnosis of implant sites. The usefulness of CT for implant dentistry was evident. However, access and direct discussion of information and results between the radiologist and the general dentist were limited. The use of software that could make an assessment of the alveolar bone for the implant planning provided better conditions to the implant surgery decreasing the surgical and prosthetic errors. $^{5}$ 
The Columbia Scientific, in 1988, created the first software for 3D analysis of alveolar bone anatomy from CT images. In nowadays, with the evolution of technology, scanners and software's it is possible with the advent of $C B C T$, to solve difficulties of conventional imaging devices for clinical dentistry, such as decreasing in the radiation dose absorbed, approximately $12.0 \mathrm{mSv}$ (microssieverts), 40 to 60 times lower than conventional $\mathrm{CT}{ }^{6}$ decreased image distortion, only $0.1 \mathrm{~mm}$ compared to 1.0 to $1.5 \mathrm{~mm}$ distortions of conventional radiographs, clinical data growth and decreased scan time. ${ }^{7}$

$\mathrm{K}$ obayashi et $\mathrm{l}^{8}$ evaluated the accuracy of cone beam tomography in cadaveric jaws and the authors concluded that $1.4 \%$ of CBCT images analyzed showed slightly unrealistic measures. This study also reported that these slightly variations were 0.01 to $0.65 \mathrm{~mm}$, not affecting the rehabilitation planning with osseointregrated dental implants. ${ }^{8}$

Due to it's superior accuracy compared to other radiological techniques, CT is essential for the advanced surgical guides fabrication that allow the analysis of threedimensional images, facilitating the choice of appropriate implants for surgery and respecting the anatomical limits of the jaws, such as: lingualfossa, mandibular canal and maxillary sinus, and to establish the correct inclination of the implants improving prosthetic result. ${ }^{5}$ Thus, from the anatomical 3D image generated by the $\mathrm{CT}$, it is possible to visualize the prosthesis to the patient and to proceed with the ideal 3D placement of the implants in accordance with the prosthetic and biomechanical requirements. ${ }^{9}$

The predictability of the long-term success of rehabilitation with dental implants depends on an accurate preoperative diagnosis. In this context guided surgery for implant placement allows conducting a reverse planning of rehabilitation, starting from the prosthesis to the surgery. ${ }^{5} \mathrm{~A}$ fter the prosthetic planning, it is possible to create a surgical guide, which aims to transfer accurate information provided by the tomographic image and analyzed in software. They can be made manually or by CAD/CAM system (computer-aided design/computer-aided manufacturing). ${ }^{9}$

Studies have shown that surgical guide allows an accuracy of less than $1 \mathrm{~mm}$ at the apex of the implant and a good control of the perforation angle compared to the radiographic guide, but the authors did not assess the change in the angle to the prosthetic settling. ${ }^{10,11}$ Other study ${ }^{12}$ analyzed the accuracy of guided surgery technique to implant placement. The authors showed an average linear deviation of $1.07 \mathrm{~mm}$ on the platform of some implants, $1.63 \mathrm{~mm}$ deviation in the apical region and $5.2^{\circ}$ in the deviation angle, but reported difficulty with the accuracy of this variations because there was not a consistently define related to the direction of deviations. ${ }^{12}$

Surgical guides are produced for imaging evaluation and surgical placement of the dental implants, increasing the outcome predictability. Surgical guides should present some basic characteristics such as: stability and stiffness, and being stable in the edentulous or partial edentulous arch. They should allow being adjusted and the size and transparency are also important aspects. ${ }^{1}$

The guides properly fixed at the surgical site, allow the realization of the sequenced implant perforations followed by implant placement in an ideal position, without any kind of movement favoring the final surgical and prosthetic outcomes. During the surgery, is used a sequence of drilling guides for each drills diameter in accordance with the virtual model planning avoiding a number of variables that could interfered with the rehabilitation successful. The accuracy of priorimplant placement and prosthetic planning allows the installation of prosthesis immediately to surgery because the positioning of the implants is identical to the virtual model. ${ }^{5}$

A detailed analysis of the patients must be performed prior to the guided surgery indication. The analysis should evaluate the presence and amount of keratinized gingiva, availability of bone height and width, necessity of bone grafts and socioeconomic conditions of the patient. ${ }^{4}$ The guided surgery is indicated for partially or completely edentulous patients, bothin the maxilla and mandible. ${ }^{3}$

A nother study evaluated 27 maxillary edentulous patients that have been rehabilitated using the guided surgery. The results showed that all patients were immediately rehabilitated 1 hour after surgery. After 1 year of followup, all the 164 implants placed were stable, with $100 \%$ of success rate. ${ }^{9}$

On the other hand, one of the possible problems that may occur using the guided surgery are the smaller deviations of the implant placement that can compromise the installation of previously planned prosthesis for immediate loading even when these deviations are smaller than conventional techniques. One study found $9.1 \%$ of preprosthetic complications and $18.8 \%$ of complications in prosthesis installation, in $12 \%$ of cases (108 patients). ${ }^{12}$ However, positive results were also found in another study, that showed reduction of standard deviation with consequent decrease in surgical errors, with the improvement in the coronal osteotomy from 1.5 to $0.9 \mathrm{~mm}$, apical positioning improvement and better angulation from $8^{\circ}$ to $4.5^{\circ} .{ }^{13}$

The most commonly complications in guided surgery are: incorrect perforation of bone tissue, implants loss, maladjustment of prosthetic components and prosthesis 
loss. These complications may be the result from errors in the various stages of surgical preparation that involves imaging scanning, surgical guideplanning, and even their fixation during the site preparation.

A nother disadvantage of this technique is the treatment cost. In all cases the cost is greater than a common surgery due to the necessity for more careful planning using other technologies. However, the high cost is viewed favorably by patients due to the various benefits of flapless surgical technique and prosthetic outcomes predictability.

\section{DISCUSSION}

The increased use of dental implants and the diminution of operative costs are providing the development and improvement of various surgical techniques used for different situations. Virtually guided surgery is a procedure that has been used with great success rate, because this technique has several advantages, including the achievement of a fast and flapless surgery.

B erdougo et al ${ }^{14}$ compared the implant surgery with the conventional technique and the flapless technique. In 169 patients were installed a total of 552 implants, where 281 implants were installed using the conventional technique and 271 implants using the flapless technique. During a period of 1 to 4 years, the patients were evaluated annually and no statistically significant difference between the two procedures were found with a success rate of $98.57 \%$ for conventional surgery and $96.30 \%$ for virtually guided surgery.

A systematic review ${ }^{15}$ evaluated 28 studies which used virtually guided surgery for the dental implants installation. A total 4032 implants were placed through this technique in 852 patients, ranging in 16 to 92 years age, with 1 to 49 months follow-up. Of the 4032 implants installed, only 167 had complications, where $64 \%$ of these complications were related to the implants and $36 \%$ in the prosthesis. The rate of implant loss was only $3 \%$.

One of the possible causes of implant loss is the inflammation followed by an infection, ${ }^{16}$ and this inflammation can be caused by any bacteremia. This bacteremia may occur at the time of the dental implants installation when blood vessels are exposed and in contact with the oral environment. ${ }^{17}$

A previous study ${ }^{17}$ evaluated the presence of bacteremia after installation of 377 dental implants in 68 patients comparing conventional technique with flapless technique. The authors analyzed the peri-implant inflammatory response and the presence of bacteria in blood samples. This study found that $62.06 \%$ of the patients treated by the conventional technique had bacteremia, while $11.76 \%$ of the patients treated by flapless surgery showed signs of bacteremia. B esides the flapless technique, the authors suggested that the reduced operatory time could be an advantage, decreasing the chance of bacteremia.

A $n$ important issue that can affect the success or failure and esthetic of the implants are the quality of the periimplant tissue around the implants. Law et $a^{18}$ evaluated implants placed in the dog mandibles through conventional and flapless surgery. The authors analyzed the peri-implant tissue after 8 weeks, and showed a less pronounced gingival recession in implants installed with flapless surgery. A lso, they observed an increased inflammation in the peri-implant tissues of implants placed with conventional surgery, evidenced by the presence of higher amount of gingival crevicular fluid, an indicator of peri-implant inflammation.

\section{CONCLUSION}

The guided surgery technique represents an advance in the field of implantology. It is indicated for several cases in oral rehabilitation with dental implants, allowing for minimally invasive surgery, decreased errors, and increased prosthesis predictability, showing more accuracy compared to the conventional surgery, despite the need of new technologies and advanced planning.

\section{REFERENCES}

1. Orentlicher G, Goldsmith D, A bboud M. Computer-guided planning and placement of dental implants. A tlas Oral M axillofacial Surgery Clinics of N orth A merica 2012;20(1): 53-79.

2. Turkyilmaz I, Suarez JC. An alternative method for flapless implant placement and an immediate provisional crown: a case report. J Contemp Dent Prac 2009;10(3):89-95.

3. deAImeida EO, Pellizzer EP, G oiatto M C, M argonar R, Rocha $E P$, Freitas AC, Jr, A nchieta RB. Computer-guided surgery in implantology: review of basic concepts. J Craniofac Surg 2010;21(6):1917-1921.

4. A zari A, Nikzad S. Flapless implant surgery: review of the literature and report of 2 cases with computer-guided surgical approach. J Oral M axillofacial Surgery: Official Journal A merican Association of Oral and M axillofacial Surgeons 2008;66(5):1015-1021.

5. Orentlicher $G, A$ bboud M . Guided surgery for implant therapy. Oral and Maxillofacial Surgery Clinics of North A merica 2011;23(2):239.

6. Clark DE, Danforth RA, Barnes RW, Burtch ML. Radiation absorbed from dental implant radiography: a comparison of linear tomography, CT scan, and panoramic and intraoral techniques. J Oral Implant 1990;16(3): 156-164.

7. Hashimoto K, Kawashima S, A raki M, Iwai K, Sawada K, A kiyama $Y$. Comparison of image performance between cone-beam computed tomography for dental use and four-row multidetector helical CT. J Oral Sci 2006;48(1): 27-34. 
8. Kobayashi K, Shimoda S, Nakagawa $Y$, Yamamoto A. A ccuracy in measurement of distance using limited cone-beam computerized tomography. The International J ournal of Oral and M axillofacial Implants 2004;19(2):228-231.

9. van Steenberghe $D, G$ lauser R, Blomback $U$, A ndersson M, Schutyser F, Pettersson A, Wendelhag I. A computed tomographic scan-derived customized surgical template and fixed prosthesis for flapless surgery and immediate loading of implants in fully edentulous maxillae: a prospective multicenter study. Clinical Implant Dentistry and Related Research 2005;7 Suppl 1:S111-120.

10. Naitoh M, A riji E, Okumura S, Ohsaki C, Kurita K, Ishigami T. Can implants be correctly angulated based on surgical templates used for osseointegrated dental implants? Clinical O ral Implants Research 2000;11(5):409-414.

11. Besimo CE, Lambrecht JT, Guindy JS. A ccuracy of implant treatment planning utilizing template-guided reformatted computed tomography. Dento Maxillofacial Radiology 2000;29(1):46-51.

12. Schneider $D, M$ arquardt $P$, Z wahlen $M$, J ung RE. A systematic review on the accuracy and the clinical outcome of computerguided template-based implant dentistry. Clinical Oral Implants Research 2009;20 Suppl 4:73-86.
13. Penarrocha $M$, B oronat A, Carrillo C, Albalat S. Computerguided implant placement in a patient with severe atrophy. The J ournal of O ral Implantology 2008;34(4):203-207.

14. B erdougo $M$, Fortin T, B lanchet $E$, Isidori $M$, Bosson J L. Flapless implant surgery using an image-guided system. A 1- to 4-year retrospective multicenter comparative clinical study. Clinical Implant Dentistry and Related Research 2010;12(2):142-152.

15. Hultin M, Svensson KG, Trulsson M. Clinical advantages of computer-guided implant placement: a systematic review. Clinical Oral Implants Research 2012;23 Suppl 6:124-135.

16. Guncu GN, Buyuktepe G, A skin SB, Kilinc K, Tumer C, Tozum TF. Inpatient evaluation of periodontal, esthetic and inflammatory parameters around dental implants and natural teeth. J Contemp Dent Prac 2013;14(1):90-95.

17. A risan V, Bolukbasi N, Oksuz L. Computer-assisted flapless implant placement reduces the incidence of surgery-related bacteremia. Clinical Oral Investigations 2012.

18. Lei Q, Chen J, Jiang J, Fu X, Lin H, Cai Z. Comparison of soft tissue heal ing around implants in beagle dogs: flap surgery versus flapless surgery. Oral Surgery, Oral M edicine, Oral Pathology and Oral Radiology 2013;115(3):e21-27. 